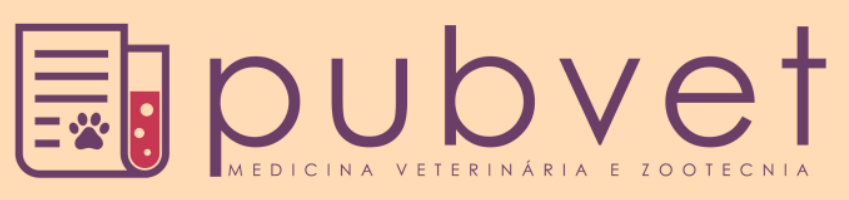

ISSN $1982-1263$

https://doi.org/10.22256/pubvet.v12n4a79.1-7

\title{
A geriatria canina e o manejo das doenças neoplásicas: Revisão
}

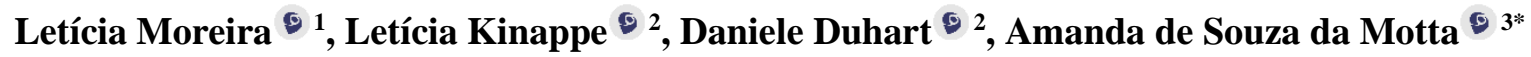 \\ ${ }^{1 *}$ Médica Veterinária Residente do Hospital de Clínicas Veterinárias da Universidade Federal do Rio Grande do Sul, Porto Alegre, RS, Brasil \\ ${ }^{2}$ Discente em Medicina Veterinária da Faculdade de Veterinária da Universidade Federal do Rio Grande do Sul, Porto Alegre, RS, Brasil \\ ${ }^{3}$ Docente do Instituto de Ciências Básicas da Saúde da Universidade Federal do Rio Grande do Sul, Porto Alegre, RS, Brasil. \\ *Autor para correspondência: Endereço: Rua Sarmento Leite, 500, sala 216 CEP: 90050-170, Centro, Porto Alegre, RS, Brasil. E-mail: amanda.motta@ ufrgs.br
}

RESUMO. Algumas mudanças de hábitos da população têm repercutido de forma importante em relação aos cuidados com animais de companhia. Nota-se uma maior aproximação proprietário - animal, o que tem feito com que os cães e gatos sejam incorporados no âmbito familiar. Da mesma forma, a Medicina Veterinária vem se desenvolvendo nas mais diferentes áreas, e a oncologia veterinária vem se destacando com as novas abordagens. As neoplasias, de forma geral, exibem considerável variação em seu aspecto semiológico, clínico e histológico e por estas razões, as previsões de comportamento dos tumores e das respostas ao tratamento podem ser extremamente difíceis e subjetivas. $\mathrm{O}$ fato de os cães estarem atingindo a maior idade tem predisposto ao aparecimento destas enfermidades com maior frequência, sendo possível o estudo destas etiologias com maior detalhamento. Logo esta revisão busca aprofundar questões que se relacionam a geriatria canina e as consequências da maior idade dos cães com o aparecimento das neoplasias. Serão abordados os aspectos relevantes sobre a etiologia das neoplasias, as suas principais complicações e o manejo das mesmas considerando as novas terapias hoje adotadas, bem como a relação observada entre os proprietários e seus animais de companhia.

Palavras chave: cães idosos, doenças infecciosas, neoplasias, quimioterapia

\section{Canine geriatrics and management of neoplastic diseases: Review}

ABSTRACT: Some changes in population habits have had an important impact on pet care. A greater approximation of owner-animal is observed, which has made dogs and cats incorporated into the family. In the same way, Veterinary Medicine has been developing in the most different areas, and veterinary oncology has been emphasizing with the new approaches. Neoplasms, in general, exhibit considerable variation in their semiologic, clinical and histological aspects and for these reasons, predictions of tumor behavior and responses to treatment may be extremely difficult and subjective. The fact that the dogs are reaching the greater age has predisposed to the appearance of these diseases with more frequency, being possible the study of these etiologies with more detail. Therefore, this review seeks to deepen questions related to canine geriatrics and the consequences of the greater age of dogs with the appearance of neoplasms. The relevant aspects on the etiology of neoplasms, their main complications and their management will be approached, considering the new therapies adopted today, as well as the observed relationship between the owners and their pets.

Keywords: elderly dogs, infectious diseases, neoplasms, chemotherapy 


\title{
La geriatría canina y el manejo de las enfermedades neoplásicas: Revisión
}

\begin{abstract}
RESUMEN: Algunos cambios de hábitos de la población han repercutido de forma importante en relación a los cuidados con animales de compañía. Se nota una mayor aproximación propietario - animal, lo que ha hecho que los perros y gatos sean incorporados en el ámbito familiar. De la misma forma, la Medicina Veterinaria se viene desarrollando en las más diferentes áreas, y la oncología veterinaria viene destacándose con los nuevos abordajes. Las neoplasias, de forma general, exhiben considerable variación en su aspecto semiológico, clínico e histológico y por estas razones, las predicciones de comportamiento de los tumores y de las respuestas al tratamiento pueden ser extremadamente difíciles y subjetivas. El hecho de que los perros estén alcanzando la mayor edad ha predispuesto a la aparición de estas enfermedades con mayor frecuencia, siendo posible el estudio de estas etiologías con mayor detalle. Esta revisión busca profundizar cuestiones que se relacionan a la geriatría canina y las consecuencias de la mayor edad de los perros con la aparición de las neoplasias. Se abordarán los aspectos relevantes sobre la etiología de las neoplasias, sus principales complicaciones y el manejo de las mismas considerando las nuevas terapias hoy adoptadas, así como la relación observada entre los propietarios y sus animales de compañía.
\end{abstract}

Palabras clave: perros añosos, enfermedades infecciosas, neoplasias, quimioterapia

\section{Introdução}

A incidência de neoplasias em animais de companhia tem aumentado nos últimos anos. Este aumento tem ocorrido devido a um número variado de razões, sendo uma das principais, a atual maior longevidade dos animais (Rossetto et al., 2009). Apesar da evolução que a oncologia veterinária tem sofrido nos últimos tempos, sabese que, aproximadamente metade dos pacientes oncológicos acabará por ser vitimada por esta doença e, a maior parte, necessitará de terapia para controle da sintomatologia e da dor (Miller et al., $\underline{2016}$ ).

Tal como no homem, um animal com doença oncológica não sofre apenas com o tumor e a sua localização, mas também com vários problemas subjacentes, como as síndromes paraneoplásicas e o stress compartilhado com a família. As síndromes paraneoplásicas são alterações induzidas pelo tumor que provocam efeitos sistêmicos significativos, conduzindo a uma piora na condição geral do paciente (Silva, 2006). Além disso, a função imunológica de cães com neoplasias é alterada, o que provavelmente aumenta a incidência de complicações infecciosas vistas neste grupo de pacientes. A presença de doenças crônicas também compromete a imunidade destes animais. Outro aspecto que contribui para esse efeito imunossupressor é a apresentação de uma neutropenia severa (Tizard, 2002).
As pessoas têm hoje o animal como um integrante da família, isto o faz merecedor de uma maior atenção e cuidado, fazendo com que sua sobrevida seja maior. Esta maior longevidade dos cães de companhia tem contribuído para o aumento do surgimento dos casos de doenças neoplásicas e suas implicações clínicas. Neste contexto, essas patologias estão podendo ser mais estudas e pesquisadas, buscando-se suas etiologias considerando: idade, raça, alimentação, medicações e hormônios já administrados e outros fatores que possam estar predispondo ao desenvolvimento das diferentes neoplasias.

\section{Geriatria canina}

A geriatria é um ramo da Medicina Veterinária voltado aos animais de maior idade, que já passaram por $75 \%$ de sua expectativa de vida. Como bem sabemos os cães menores tem uma expectativa de vida maior, atingindo a faixa geriátrica em uma idade mais avançada (a partir dos 11,5 anos). Já os cães maiores a atingem um pouco antes, variando o tempo de acordo com seu peso corporal (início de 7,5 a 10,9 anos). Somente o envelhecimento não é uma doença. Este é sim, um processo fisiológico de redução progressiva das funções biológicas (De Nardi et al., 2002). Podemos citar como alguns dos principais efeitos da idade em cães e gatos a alteração na capacidade auditiva, mudanças de comportamento de micção e higiene, mudanças nos hábitos alimentares, problemas respiratórios, alterações da visão, 
mudanças no peso (aumento ou diminuição), alteração no padrão sono-vigília e cansaço.

Uma característica comum dos sistemas orgânicos senis é a alteração progressiva e irreversível. Os animais idosos raramente possuem uma única doença, e sim uma combinação particular de múltiplas doenças orgânicas com níveis variados de disfunção. Sendo assim, o conhecimento das alterações patológicas comuns associadas com a idade e seus efeitos sobre as funções vitais, permite que o médico veterinário planeje a abordagem adequada para cães e gatos idosos. A tabela 1 descreve os efeitos físicos e metabólicos comuns do envelhecimento (Hoskins, 2008).

Tabela 1. Efeitos do envelhecimento em animais de companhia

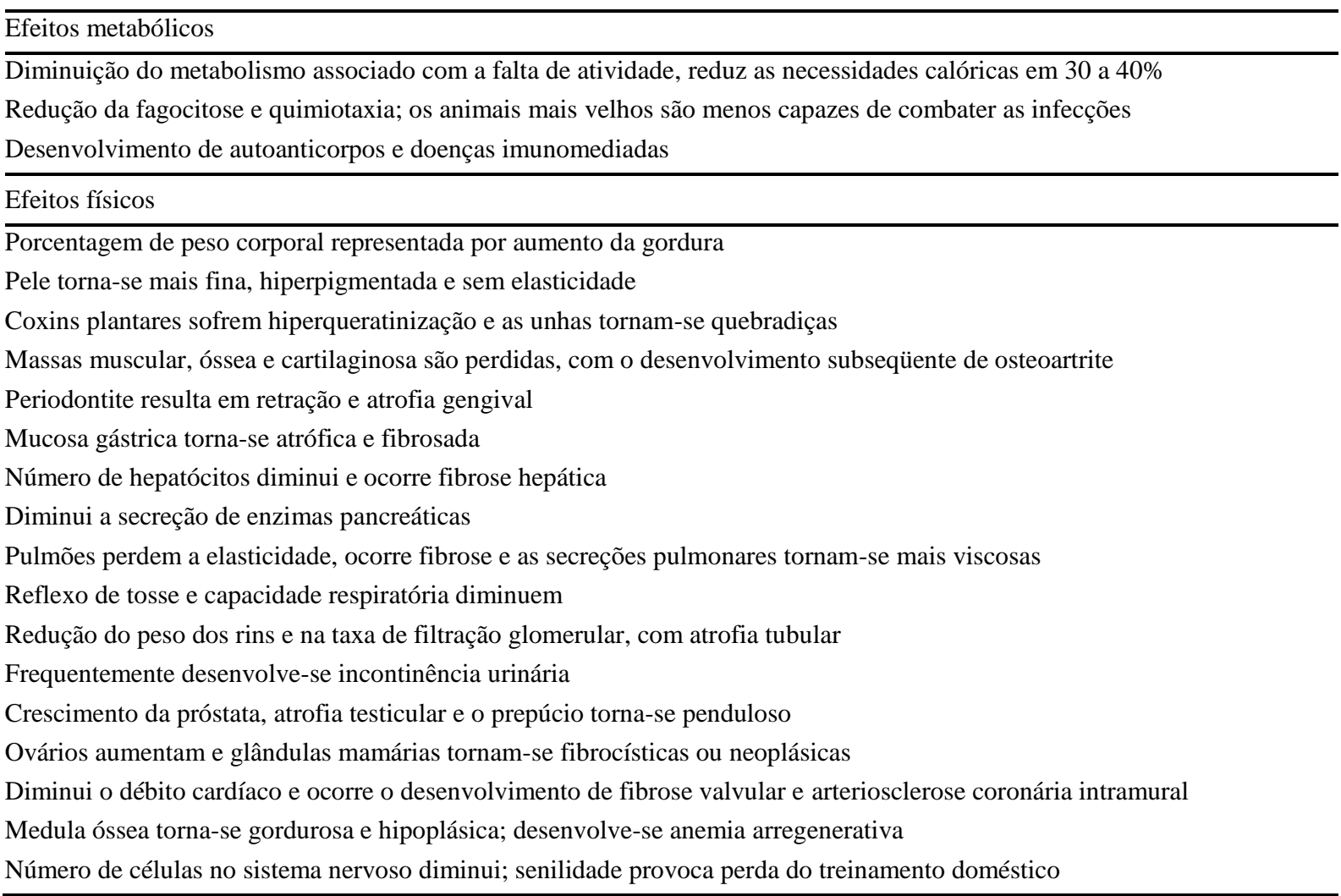

\section{Neoplasias em cães geriatras}

O envelhecimento representa o único fator de risco bem conhecido para o desenvolvimento do câncer. A idade avançada está associada com o aumento da incidência de cânceres benignos e malignos (Hoskins, 2008). Dados epidemiológicos apontam que em cães a incidência de câncer é de aproximadamente $1 \mathrm{em}$ cada 3 e em gatos esta relação é de 1 caso para 45 (Leandro \& Sá, 2016). Porém, ainda há uma grande necessidade de reconhecimento do câncer e sua relação com doenças crônicas; uma causa de alto índice de mortalidade em pequenos animais (Argyle \& Blacking, 2008).

As neoplasias em animais na faixa geriátrica podem ser mais difíceis de diagnosticar, pois os sintomas do início da doença são mascarados por outros problemas apresentados nesta faixa etária, como: artrite, obesidade, doença periodontal, entre outras (Andrade, 2008). Animais idosos requerem uma observação mais criteriosa e consultas mais frequentes para detectar o possível início de qualquer neoplasia. Neoplasias malignas são achados frequentes em animais de estimação geriatras. Esta é frequentemente uma condição limitadora. Atenção especial deve ser dada as seguintes neoplasias: carcinoma de células escamosas, melanoma, fibrossarcoma, osteossarcoma, mastocitoma, hemangiopericitoma, adenocarcinoma mamário, linfossarcoma e carcinoma vesical e uretral (Epstein et al., 2005).

Conforme relatado por Rocha et al. (2010) foi atendido no Hospital Veterinário da FAMED Garça - SP, um cão com queixa principal de 
claudicação acentuada nos membros posteriores, além de fezes amolecidas e hiporexia. Ao exame físico notou-se aumento de volume em região de crista ilíaca direita, o exame citológico dessa massa foi compatível com carcinoma de células escamosas. O prognóstico desse tipo de neoplasia é reservado, sendo a taxa de recorrência alta quando o tumor é marginalmente extirpado. Esse relato de caso evidencia que mesmo com protocolos quimioterápicos antineoplásicos, são poucos os casos de cura, devendo-se avaliar o quanto se pode estender com qualidade de vida a sobrevida do paciente, para que se possa decidir até quando prosseguir com o tratamento.

\section{Fatores de risco e prevenção do câncer}

As causas primárias do câncer ainda não estão totalmente elucidadas, mas sabe-se que as neoplasias surgem em decorrência de mutações genéticas espontâneas ou induzidas por agentes patogênicos. Os fatores causadores do câncer podem ser divididos em micro e macroambientais, também identificados como intrínsecos e extrínsecos.

Os fatores extrínsecos compreendem metais, radiações, vírus, bactérias e radicais livres de oxigênio, inflamações crônicas e xenobióticos. Já os fatores intrínsecos são representados pela idade, tipo de dieta, efeitos hormonais e predisposição genética (Daleck et al., 2008).

Atualmente o câncer é a principal causa de óbito em cães e gatos. É provável que a alta prevalência das doenças malignas na espécie canina e felina esteja correlacionada à maior longevidade desses animais, além do crescente aumento populacional (Daleck et al., 2016). Entende-se que quanto mais o animal vive, maior a exposição do organismo aos agentes carcinógenos do meio ambiente ou à combinação com os fatores intrínsecos. Assim aumenta-se a probabilidade de o organismo acumular alterações moleculares responsáveis pela multiplicação celular desordenada (Morimoto et al., 2017).

\section{Abordagens terapêuticas nas neoplasias}

Os tratamentos de neoplasias e a sua especialização têm aumentado significativamente nos últimos 20 anos. As informações disponibilizadas aos clínicos, e por consequência aos tutores de cães, fazem com que as abordagens terapêuticas sejam mais entendidas e de maior aceitação empregando, assim, melhor tratamento e condição de vida ao paciente. Disponibilizam-se maiores opções e acesso a medicamentos e equipamentos, melhorando o controle da neoplasia e seus efeitos secundários (Morimoto et al., 2017).

Para escolher o protocolo de tratamento da neoplasia, devem-se considerar alguns fatores, como custo, estado do animal, doenças concomitantes e envolvimento do proprietário. Dentre os possíveis tratamentos estão cirurgia, radioterapia e quimioterapia, sendo que para animais idosos deve-se adotar uma terapêutica conservativa, uma vez que suas reservas fisiológicas são menores, elevando as possibilidades de toxicidade dos antineoplásicos (Hoskins, 2008).

O objetivo da cirurgia oncológica visa promover a remoção de todas as células tumorais, sendo considerada um tratamento definitivo para os tumores sólidos, solitários e de baixo grau. Além de tratamento, a cirurgia ainda é importante como diagnóstico terapêutico e na citorredução da massa tumoral antes da radioterapia e tratamento definitivo para tumores sólidos, solitários, de baixo grau. $\mathrm{O}$ tratamento cirúrgico não é efetivo em casos de neoplasias disseminadas, sendo a quimioterapia indicada nesses casos (Morris, 2007).

A radioterapia recomendada para idosos deve incluir a individualização dos protocolos de tratamento, considerando número de sessões, duração e dose total. Uma das desvantagens da radioterapia é a necessidade de anestesiar o animal em cada sessão, o que pode gerar complicações em animais mais velhos (Hoskins, 2008).

As abordagens quimioterápicas na área humana já vêm sendo praticadas por muitos anos, com uma evolução importante quanto às medicações empregadas. Hoje observa-se uma maior eficiência dos tratamentos sejam eles isolados ou associados com outras práticas como a radioterapia, tendo-se ainda observado uma redução dos efeitos adversos ou colaterais, advindos dos tratamentos prolongados. Ao longo dos últimos anos a oncologia veterinária também sofreu incrementos, tendo-se tornado uma prática em algumas clínicas veterinárias. Porém a partir destas abordagens sabe-se das consequências destes processos sobre os animais de companhia levando-os a uma redução da imunidade tornandoos suscetíveis a outros processos como as doenças infecciosas. 
Dentre os efeitos das drogas quimioterápicas temos a redução da imunidade destes pacientes o que faz com que a administração das medicações deva ser feita com cautela. A mielossupressão é uma consequência grave. Há necessidade de que hemogramas sejam feitos, de modo a controlar o grau de toxicidade hematológica, tendo-se uma recomendação segundo alguns autores (Cirillo, 2008, Nelson \& Couto, 2015) de que a primeira avaliação seja feita antes de casa sessão. O segundo deve ser realizado no momento do nadir (tempo entre a administração da droga e o aparecimento do menor valor de contagem hematológico) com o intuito de avaliar o risco de neutropenia séptica. Em geral, este efeito é constatado com a maioria das drogas utilizadas em oncologia veterinária uma semana após o início do tratamento, embora possa existir variação entre pacientes. Isso vai depender também da droga usada, dosagem, modo de administração e tempo decorrido desde o último tratamento. A neutropenia normalmente ocorre por três a cinco dias, sendo considerada a fase mais perigosa do ciclo (Souza et al., 2008). Logo, exames devem ser realizados e o paciente acompanhado, pois caso haja uma contagem de leucócitos baixa, ou seja, inferior a 4.000 células/ $\mu \mathrm{L}$, ou uma contagem de neutrófilos inferior a 2.500 células/ $\mu \mathrm{L}$, ou ainda se a contagem de plaquetas diminuir para menos de $50 \times 10^{3}$ células $/ \mu \mathrm{L}$ o tratamento deve ser interrompido e apenas retomado quando o número de células sanguíneas apresentarem-se normais (Cirillo, 2008, Souza et al., 2008, Nelson \& Couto, 2015).

Entre as modalidades de cirurgia oncológica a criocirurgia é bastante utilizada em casos específicos em medicina veterinária. Embora seja tradicionalmente mais aplicada na dermatologia em lesões pré-malignas e nas neoplasias benignas e malignas, hoje as aplicações se ampliaram para diferentes neoplasias em outros órgãos como olhos, próstata, fígado, pâncreas, pulmão, linfonodos, ossos e sistema nervoso. A crionecrose é a lesão celular direta, em resposta à exposição à temperatura negativa extrema. Outro mecanismo é a lesão vascular. O primeiro evento da crioterapia ocorre com o congelamento da água contida nos tecidos, que forma cristais extracelulares e intracelulares dependendo da velocidade de congelamento e em seguida ocorre o descongelamento. Quando o congelamento ocorre rapidamente, formam-se cristais intracelulares e na sequência do descongelamento lento, os pequenos cristais inicialmente inócuos se transformarão em grandes cristais, que serão deletérios para a célula. Os agentes criogênicos são gases que podem ser convertidos ao estado líquido e têm capacidade de extrair calor de tecidos vivos. Diferentes gases atingem diferentes temperaturas e pontos de ebulição, e estas características ditam sua adequação ao uso na criocirurgia, pois o tecido-alvo deve chegar rapidamente a no mínimo $-20^{\circ} \mathrm{C}$. O agente de escolha é o nitrogênio líquido por ter ponto de ebulição extremamente baixo $\left(-195,8^{\circ} \mathrm{C}\right)$ e ser efetivo em lesões benignas e malignas ( al., 2008).

Outra possibilidade de tratamento atualmente é a eletroquimioterapia que consiste de um processo de quimioterapia potencializada pela eletroporação da membrana citoplasmática por meio de pulsos elétricos. Tem sido empregada como forma de tratamento, único ou coadjuvante para tumores superficiais como carcinomas espinocelulares, melanomas orais e mastocitomas (Brunner, 2016).

A terapia fotodinâmica, outra alternativa no tratamento de neoplasias, é uma modalidade terapêutica que envolve um fotossensibilizador (FS), luz e oxigênio molecular. O FS é ativado por determinado comprimento de onda causando uma instabilidade no fármaco que reage diretamente com o oxigênio molecular, levando à espécies reativas de oxigênio, capazes de destruir todo o tecido onde a reação esteja acontecendo (Rocha et al., 2016).

A terapia de suporte também se faz de grande importância no tratamento, principalmente quando nos referimos a pacientes idosos. É necessário monitorar a ingestão de alimentos, o consumo de água e eliminação de urina e fezes dos animais idosos, iniciando uma terapia ou um estímulo adequado quando necessário. A dor pode ser tratada de inúmeras formas, incluindo aspirina, corticosteroides, anti-inflamatórios não esteroidais e até mesmo acupuntura, sendo um cuidado essencial para determinar qualidade de vida para esses pacientes (Hoskins, 2008).

\section{Complicações em pacientes com neoplasias}

Uma das complicações associadas com o aparecimento das doenças neoplásicas são as doenças infecciosas, para as quais alguns fatores de risco podem ser relacionados. Dentre eles observam-se: a idade avançada dos pacientes, o gênero feminino como sendo mais afetado, o uso de cateteres venosos, pacientes hospitalizados, 
presença de outras doenças como diabetes, insuficiência renal, pacientes transplantados, feridas cirúrgicas, aspectos nutricionais (caquexia), neutropenia, hipotermia, hipotensão, traqueostomia, outras doenças crônicas e progressivas, quimioterapia prolongada, instrumentalização inadequada, ascite e outros (Martins et al., 2009). Considerando a presença de uma doença infecciosa associada a um processo de neoplasia, a primeira atitude a ser tomada trata-se da identificação do agente etiológico envolvido, aliado a uma anamnese minuciosa. $\mathrm{O}$ passo seguinte refere-se à avaliação do grau de suscetibilidade deste agente aos antibióticos empregados rotineiramente, isto de modo a evitar a administração de antibióticos contra os quais a bactéria já apresente um perfil de resistência.

Em relação à presença de infecções em pacientes humanos, a bacteremia e a endocardite infecciosa por Streptococcus bovis parece estar relacionada com a presença de lesões neoplásicas no intestino grosso e doenças hepáticas. Há um trabalho com o relato de que pacientes com endocardite infecciosa por $S$. bovis e colonoscopia normal, podem ser incluídos como um grupo de risco para o desenvolvimento de câncer de cólon (Waisberg et al., 2002).

Os pacientes oncológicos também sofrem com a neutropenia febril, choque séptico e disfunção nos órgãos e sistemas. A classificação da neutropenia febril quanto ao risco infeccioso pode auxiliar na escolha da terapia antimicrobiana, na definição da via a ser utilizada, na possibilidade de terapia em nível ambulatorial e na etiologia provável (Maschmeyer et al., 2003). Ainda, o atraso na administração do antibiótico no paciente neutropênico pode aumentar o risco de infecção. Por outro lado, o uso indiscriminado do antimicrobiano pode alterar a microbiota bacteriana no trato gastrintestinal, aumentando assim o potencial de infecção sistêmica, principalmente após lesões da camada mucosa do intestino (Cirillo, 2008).

A investigação laboratorial destes processos é de suma importância para o desenvolvimento da área clínica, por esse motivo faz-se importante estudar e pesquisar as principais bactérias ligadas ao câncer e estabelecer se possível, uma relação entre as causas para o diagnóstico, o tratamento e o prognóstico em animais, considerando a complexidades dos processos neoplásicos. A literatura traz poucas informações sobre a correlação de neoplasias em animais e suas associações com doenças de natureza infecciosa.

\section{Considerações finais}

A carcinogênese é um processo complexo no qual contribuem tanto fatores intrínsecos (predisposição herdada) quanto extrínsecos (fatores de risco ambientais). É possível que, no futuro, por meio da manipulação genética, possase alterar a predisposição herdada, mas hoje o alcance das estratégias para reduzir o risco de câncer restringe-se aos fatores de risco ambientais.

Considerando o que foi abordado sobre a presença das neoplasias em cães idosos, sobre a longevidade e a qualidade de vida dos animais de companhia, os profissionais da área veterinária devem preparar-se para um incremento nos estudos científicos de forma aplicada para a cura das enfermidades neoplásicas. A identificação de novos fármacos, bem como a avaliação de sua toxicidade são fundamentais para a eleição de um novo princípio ativo. A investigação aplicada deve prever ensaios clínicos, estudos de prognóstico, predição das respostas e estudos farmacodinâmicos em amostras biológicas obtidas de pacientes que receberam terapia antitumoral. As informações clínicas sobre enfermidade e a resposta frente à toxicidade ao tratamento são também importantes e devem, da mesma forma, ser consideradas.

\section{Referências Bibliográficas}

Andrade, S. F. 2008. Manual da Terapêutica Veterinária. Rocca, São Paulo.

Argyle, D. J. \& Blacking, T. 2008. From viruses to cancer stem cells: dissecting the pathways to malignancy. The Veterinary Journal, 177, 311323.

Brunner, C. H. 2016. Eletroquimioterapia. In: Daleck, C. R. \& De Nardi, A. B. (eds.) Oncologia em cães e gatos. Editora Roca, Sãp Paulo, Brasil.

Cirillo, J. V. 2008. Tratamento quimioterápico saúde. Revista do Instituto de Ciências da Saúde, 26, 325-327.

Daleck, C. R., De Nardi, A. B., Rodigheri, S. M. \& Motta, F. R. 2008. Neoplasia do sistema urinário. Roca, São Paulo.

Daleck, C. R., Fonseca, C. S. \& Canola, J. C. 2016. Oncologia em cães e gatos. Roca, Rio de Janeiro. 
De Nardi, A. B., Rodaski, S., Sousa, R. S., Costa, T. A., Macedo, T. R., Rodigheri, S. M., Rios, A. \& Piekarz, C. H. 2002. Prevalência de neoplasias e modalidades de tratamentos em cães, atendidos no hospital veterinário da Universidade Federal do Paraná. Archives of Veterinary Science, 7, 15-26.

Epstein, M., Kuehn, N. F., Landsberg, G., Lascelles, B. D. X., Marks, S. L., Schaedler, J. M. \& Tuzio, H. 2005. Senior care guidelines for dogs and cats. Journal of the American Animal Hospital Association, 41, 81-91.

Hoskins, J. D. 2008. Geriatria e Gerontologia do cão e gato. Editora Roca, São Paulo, Brasil.

Leandro, R. M. \& Sá, L. R. M. 2016. Tumor estromal gastrointestinal em cães: estudo clínico-anatomopatológico. Arquivo Brasileiro de Medicina Veterinária Zootecnia, 68, 4, 938944.

Martins, C. A. d. S., Faria, L. M. D. d., Souza, M. C., Camello, T. C. F., Velasco, E., Hirata Júnior, R., Thuler, L. \& Mattos-Guaraldi, A. L. 2009. Microbiological and host features associated with corynebacteriosis in cancer patients: a five-year study. Memórias do Instituto Oswaldo Cruz, 104, 905-913.

Maschmeyer, G., Ostermann, H., Wendt, S. \& Richter, G. 2003. Guidelines of the infectious diseases working party of the German Society of Hematology and Oncology. Annual Hematology, 82, S105-117.

Miller, R. L., Van Lelyveld, S., Warland, J., Dobson, J. M. \& Foale, R. D. 2016. A retrospective review of treatment and response of high- risk mast cell tumours in dogs. Veterinary and Comparative Oncology, 14, 361-370.

Morimoto, C. Y., Tedardi, M. V., da Fonseca, I. I. M., Kimura, K. C., Sanches, D. S., Epiphanio, T. F., Strefezzi, R. F. \& Dagli, M. L. Z. 2017. Evaluation of the global Dna methylation in canine mast cell tumour samples by immunostaining of 5-methyl cytosine. Veterinary and Comparative Oncology, 15, 1014-1018.

Morris, J. 2007. Oncologia em pequenos animais. Editora Roca, São Paulo, Brasil.
Nelson, R. \& Couto, C. G. 2015. Medicina interna de pequenos animais. Elsevier Brasil, São Paulo, Brasil.

Rocha, J. R., Santos, L. M., Trentin, T. C., Rocha, F. P. C., Pacheco, M. D. \& riolani, M. 2010. Carcinoma de células escamosas em cão: relato de caso. Revista Científica Eletrônica de Medicina Veterinária, 14, 1-14.

Rocha, M., Longo, J. P., Lucci, C. M. \& Azevedo, R. B. 2016. Terapia Fotodinâmica em Veterinária. In: Daleck, C. R. \& De Nardi, A. B. (eds.) Oncologia em cães e gatos. Editora Roca Ltda., Rio de Janeiro, Brasil.

Rossetto, V. J. V., Moreno, K., Grotti, C. B., Reis, A. C. F. \& Bracarense, A. P. F. R. L. 2009. Frequência de neoplasmas em cães diagnosticados por exame citológico: estudo retrospectivo em um hospital-escola Frequency of tumors in dogs based on cytological diagnosis: a retrospective study in a veterinary teaching hospital. Semina: Ciências Agrárias, Londrina, 30, 189-200.

Silva, M. P. N. 2006. Síndrome da anorexiacaquexia em portadores de câncer. Revista Brasileira de Cancerologia, 52, 59-77.

Souza, W. A., Negrão, A. J. M., Filho, C. G., Ferreira, V. H., Lino, R. S., Freitas, R. E., Rogério, E., Biazotto, G. \& Pereira, D. M. 2008. Toxicidade de antineoplásiscos. Revista Científica Eletrônica de Medicina Veterinária, 11, 1-6.

Tizard, I. R. 2002. Imunologia veterinária-uma introdução. Editora Roca, São Paulo, Brasil.

Waisberg, J., Matheus, C. d. O. \& Pimenta, J. 2002. Infectious endocarditis from Streptococcus bovis associated with colonic carcinoma: case report and literature review. Arquivos de Gastroenterologia, 39, 177-180.

Article History:

Received 3 January 2018

Accepted 1 February 2018

Available online 9 April 2018

License information: This is an open-access article distributed under the terms of the Creative Commons Attribution License 4.0, which permits unrestricted use, distribution, and reproduction in any medium, provided the original work is properly cited. 